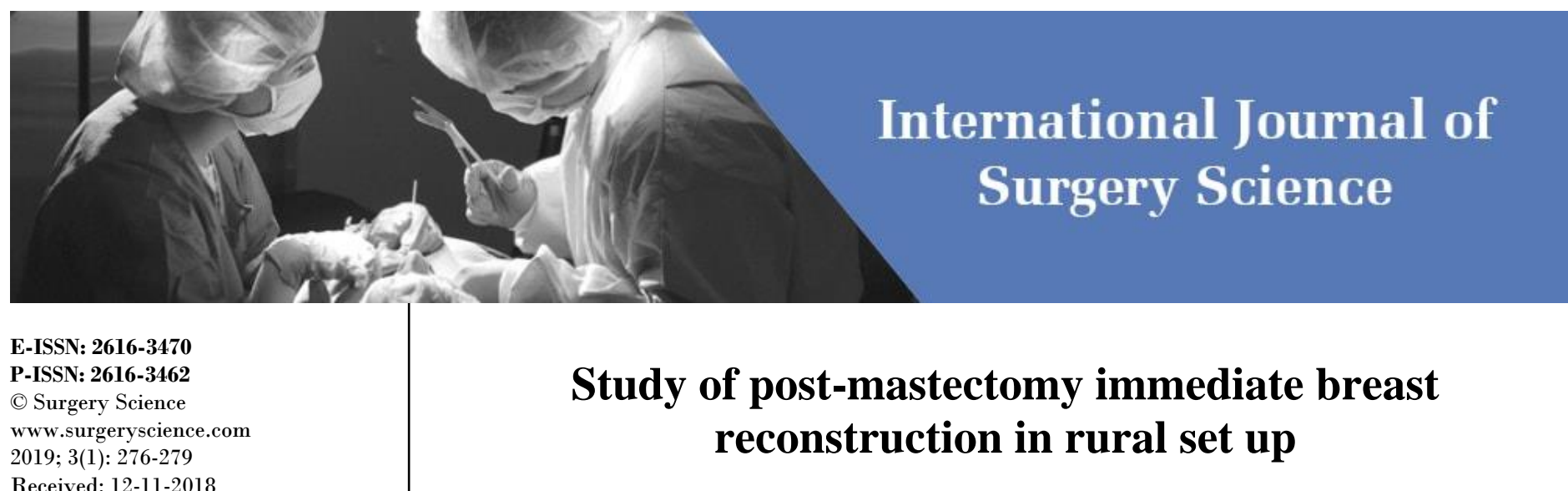

Accepted: 15-12-2018

\section{Dr. Vijay Potey}

Associate Professor; Department of Surgery, SVN Govt Medical College Yavatmal, Maharashtra, India

\section{Dr. Vijay Kanake}

Assistant Professor, Department of Surgery, SVN Govt Medical College Yavatmal, Maharashtra, India

\section{Dr. Pawan Survase}

Resident, Department of Surgery; SVN Govt Medical College Yavatmal, Maharashtra, India

\section{Correspondence}

Dr. Vijay Kanake

Assistant Professor, Department of Surgery, SVN Govt Medical College Yavatmal, Maharashtra, India

\section{Dr. Vijay Potey, Dr. Vijay Kanake and Dr. Pawan Survase}

DOI: https://doi.org/10.33545/surgery.2019.v3.i1e.47

\section{Abstract}

Background: As the number of mammary reconstruction surgeries performed has increased in recent years, reconstructive surgery of the nipple and areola has also become more widespread. This study was planned to check feasibility of Post mastectomy Immediate Breast Reconstruction in rural set up.

Methodology: Prospective observational study conducted in 30 patients with diagnosed breast malignancy. After proper evaluation, history and physical examination patient were posted for surgery. LD flap and TRAM flap surgery depending on the stage of the patient and extent of the disease were done. Patients were evaluated in the post-operative period for pain, discomfort, development of complications. All the patients were followed up for 6 months during which patient complications and satisfaction noted.

Results: Mean age of patients undergoing immediate post-operative breast reconstruction was $39.5 \mathrm{yrs}$ (2855yrs). 39.28\% patients who underwent Post Mastectomy Immediate Breast Reconstruction had stage II A of Breast Carcinoma, $35.72 \%$ patients had stage IIB, $14.28 \%$ patients had stage IIIA disease and $10 \%$ patients had stage IIIB disease. $18.18 \%$ patients who underwent LD flap reconstruction had marginal flap necrosis.

Conclusion: LD Flap reconstruction is simple to perform and safest technique of Breast Reconstruction. TRAM flap is cosmetically excellent but good only in expert hands. Nipple and areola reconstruction is final stage after immediate breast reconstruction is not mandatory and depends on patient's choice. This study concludes that Post Mastectomy Immediate Breast Reconstruction can be feasible in rural set up.

Keywords: Breast, mastectomy, reconstruction, LD flap

\section{Introduction}

Breast is the symbol of feminity, attractiveness and motherhood. Loss or disfiguration of this organ results in loss of feminine attractiveness, an altered body perception leading to intense psychosexual problems and mental depression ${ }^{[1]}$ Breast reconstruction, particularly when performed at the time of mastectomy, reduces depression and improves well-being in breast cancer survivors ${ }^{[2]}$ During the Halstedian era, which lasted for many years, radical mastectomy was the treatment offered for carcinoma breast ${ }^{[3]}$. Today the concepts have changed and it is more of Breast Conservative Surgery. This is made possible because of early detection, better investigative facilities, increased understanding of the pattern of disease spread and better forms of adjuvant treatment options ${ }^{[1]}$.

In recent years, immediate resurfacing with local arterial flaps when skin cover is inadequate and use of silicone prostheses have made possible a better and more aesthetically acceptable reconstruction ${ }^{[4]}$. Increasingly breast surgery is performed by surgeons trained in oncoplastic techniques contributing oncological and aesthetic procedures in one operation and having excellent therapeutic and economic benefits ${ }^{[3]}$. The aim of immediate breast reconstruction is restoration of the breast mound at the time of mastectomy and to create a breast, which offers softness, symmetry, sensibility, aesthetically acceptable scars, as well as texture and colour similar to the healthy side. The optimal method should offer a result that makes the patient feel as natural as possible. It should be safe, reliable, and accompanied by little donor-site morbidity. By achieving an acceptable appearance, reconstruction of the breast should improve the patient's quality of life and offer no subsequent health risk ${ }^{[3]}$ Broadly, techniques of reconstruction are available for post mastectomy immediate breast reconstruction: Tissue Expander/Implant Reconstruction; Pedicle Latissimus Dorsi (LD) Flap (With or Without an Implant) and Transfer of abdominal fat in the form of a Transverse Rectus Abdominis Myocutaneous (TRAM) Flap or a perforator flap using the same abdominal skin and fat but no muscle, based on deep inferior 
epigastric perforator vessels (DIEP). Alternative free flaps using buttock or thigh fat are offered in some centres ${ }^{[4]}$. Nipple and Areola Reconstruction is the final stage of breast reconstruction. It is commonly done after 3 months. As the number of mammary reconstruction surgeries performed has increased in recent years, reconstructive surgery of the nipple and areola has also become more widespread. This study was planned to check the feasibility of Post mastectomy Immediate Breast Reconstruction in rural set up.

\section{Materials and Methods}

Prospective observational study conducted at Shri V. N. Government Medical College, Yavatmal. Total 30 patients with diagnosed breast malignancy were selected for the study. Stage IV disease and patients not willing for the procedure were excluded from the study. After proper evaluation, history and physical examination patient were posted for surgery. Type of surgery depended on the stage of the patient and extent of the disease. Pros and cons of the procedure explained and Proper consent obtained. All patients were operated by Senior General Surgeon at our hospital. Patients were evaluated in the postoperative period for pain, discomfort, development of complications. All the patients were followed up for 6 months during which patient complications and satisfaction noted.

2.1 Latissimus Dorsi FLAP: Incision is put as shown in the figure 1 depending on the area of skin needed for the cover. The slanting placed vertical incision, along the posterior axillary fold, is deepened first to identify the muscle and then the skin flaps are raised to find the anterior border of the Latissimus Dorsi muscle which is raised off the lateral chest wall. The island of skin already marked along with the underlying tissues including the muscle is harvested to be placed to the recipient area of the mastectomy site.

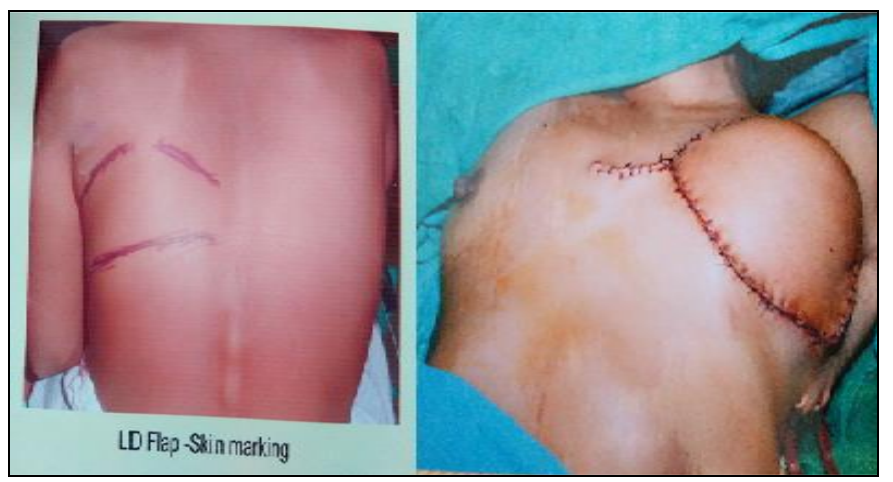

Fig 1: Pre-operative marking and post-operative photo of postmastectomy immediate breast reconstruction with LD flap.

2.2 LD flap with simple insertion of silicone implant: permanent pre filled silicone implant placed in sub-pectoral pocket.

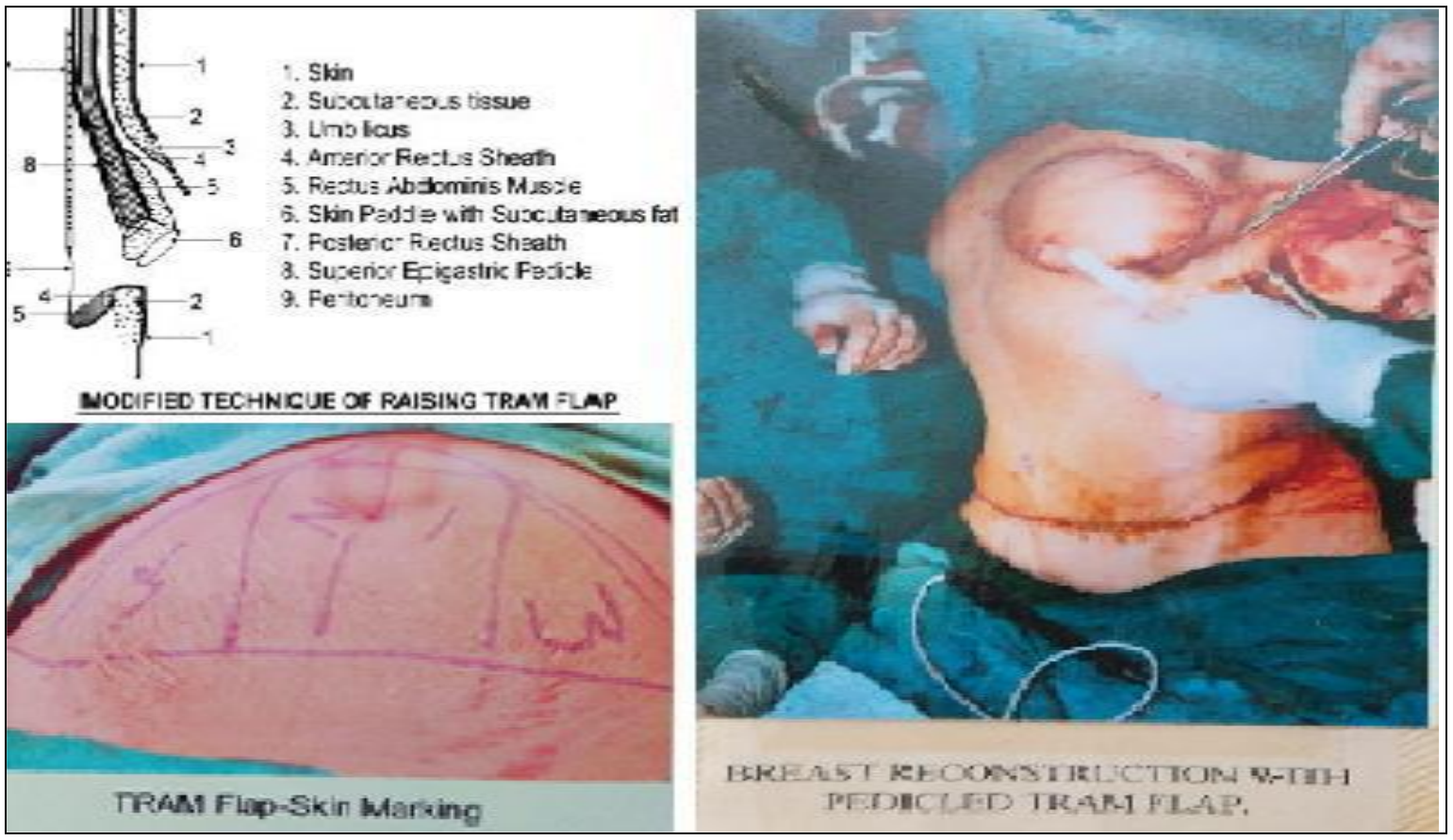

Fig 2: Photo Intra-operative photo of Silicone implant for breast reconstruction and TRAM flap pre-op marking and intra-op photo.

2.3 Tram Flap: This flap was planned based on the superior epigastric vessels. Incision extends from the anterior superior iliac spine to $1-2 \mathrm{~cm}$ lateral to the midline over the contralateral rectus Abdominis muscle. The vertical arc extends from $1-2 \mathrm{~cm}$ above the umbilicus to include the important perforators and the inferior limit is situated $3-4 \mathrm{~cm}$ above the symphysis pubis as shown in figure 3 . This area represents around $50-60 \%$ of the lower abdominal surface area. After delineation of the flap, dissection begins starting from lateral to medial (extending deeply to the abdominal wall aponeurosis) until the lateral border of the rectus Abdominis is reached. At that point, the rectus sheath is incised to expose the inferior epigastric vessels passing superiorly underneath the muscle, where they are ligated and the flap is then elevated along the plane deep to the vascular pedicle and taking the entire rectus muscle and overlying rectus sheath with the skin island. The flap is then tunneled beneath the abdominal wall upwards till the xiphoid process where the superior epigastric vessels are carefully exposed; they usually enter the muscle $2-3 \mathrm{~cm}$ lateral to the midline. The rectus muscle is severed from the xiphoid attachment to allow for flap rotation and in-setting at the mastectomy bed. Final contouring of the flap is carefully carried out while the patient is in the sitting position to achieve symmetry with the normal breast Particular attention should be experienced for the location and symmetry of the inframammary sulcus. 
2.4 Nipple and areola reconstruction: A symmetric position is determined preoperatively with patient in standing position. A skin hyphen fat flap (modified skate) is elevated to create a new nipple. After nipple reconstruction graft is harvested and sutured in position. Tattooing can be used to simulate areola at time of nipple reconstruction or can be used to match contralateral.

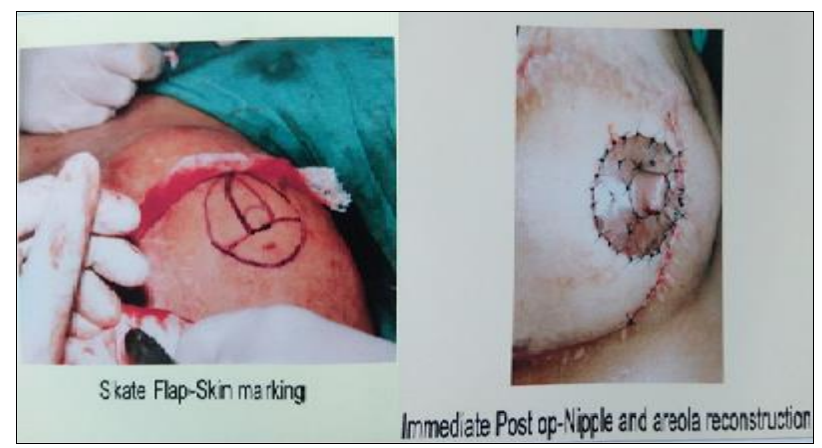

Fig 3: Technique of Nipple and Areola Reconstruction.

Total 30 patients underwent immediate post-operative breast reconstruction. Mean age of patients undergoing immediate post-operative breast reconstruction was 39.5 yrs (28-55yrs). Total 28 patients had Carcinoma Breast and 2 patients had Phylloids tumour. 39.28\% patients who underwent Post Mastectomy Immediate Breast Reconstruction had stage II A of
Breast Carcinoma, $35.72 \%$ patients had stage IIB, $14.28 \%$ patients had stage IIIA disease and $10 \%$ patients had stage IIIB disease.

Table 1: Age Characteristics of study participants

\begin{tabular}{|c|c|c|}
\hline Age (years) & No. of study participants & Percentage \\
\hline$<30$ & 3 & 10 \\
\hline $30-50$ & 19 & 63.3 \\
\hline$>50$ & 8 & 26.6 \\
\hline \multicolumn{3}{|c|}{ Stages of breast carcinoma (n:28) } \\
\hline Stage 0 & 0 & 0 \\
\hline Stage I & 0 & 0 \\
\hline Stage II a & 11 & 39.2 \\
\hline Stage II b & 10 & 35.7 \\
\hline Stage III & 04 & 14.2 \\
\hline Stage III B & 03 & 10.7 \\
\hline Stage III C & 0 & 0 \\
\hline Stage IV & 0 & 0 \\
\hline \multicolumn{3}{|c|}{ Procedure Done } \\
\hline LD flap & 16 & 53.3 \\
\hline LD flap with Implant & 06 & 20 \\
\hline TRAM flap & 08 & 26.6 \\
\hline \multicolumn{2}{|c|}{ Nipple and areola reconstruction } \\
\hline Done & 6 & 20 \\
\hline Not Done & 24 & 80 \\
\hline \multicolumn{2}{|c|}{} \\
\hline
\end{tabular}

\section{Results}

Table 2: Symmetry of Breast achieved and late complications who underwent Post Mastectomy Immediate Breast Reconstruction.

\begin{tabular}{|c|c|c|}
\hline & No. of study participants & Percentage \\
\hline \multicolumn{2}{|c|}{ Symmetry of Breast achieved after Breast Reconstruction } \\
\hline Symmetrical & 14 & 46.7 \\
\hline Asymmetrical & 16 & 53.3 \\
\hline \multicolumn{2}{|c|}{ Late Complications } \\
\hline Recurrence of carcinoma breast & 2 & 6.6 \\
\hline Abdominal Hernia & 0 & 0 \\
\hline Abdominal Laxity & 0 & 0 \\
\hline
\end{tabular}

Table 3: Comparison of duration of hospital stay and complications among study participants with different procedure

\begin{tabular}{|c|c|c|}
\hline & LD Flap (\%) & TRAM Flap (\%) \\
\hline $\begin{array}{c}\text { Duration of hospital stay } \\
\text { (days) (mean } \pm \text { SD) }\end{array}$ & $9.8 \pm 2.3$ & $12.7 \pm 3.4$ \\
\hline \multicolumn{2}{|c|}{ Complications } \\
\hline Marginal flap necrosis & $6(20)$ & $11(36.6)$ \\
\hline Partial flap necrosis & 0 & 19 \\
\hline Total flap necrosis & 0 & 0 \\
\hline Seroma & $4(13.3)$ & 0 \\
\hline Donor site infection & $3(10)$ & $4(13.3)$ \\
\hline
\end{tabular}

In Present study, $18.18 \%$ patients who underwent LD flap reconstruction had marginal flap necrosis. Partial and Total flap necrosis was not observed in any of these patients.

\section{Discussion}

Post Mastectomy Immediate Breast Reconstruction has definite advantages over mastectomy alone by providing good cosmesis. The appreciation of oncologic safety has led to Immediate Reconstruction of Breast in a single anaesthesia over the past few years. The main aim in breast reconstruction is to create a breast, which offers softness, symmetry, sensibility, aesthetically acceptable scars, as well as texture and colour similar to the healthy side.

Modified Radical Mastectomy was the primary procedure done in maximum $93.3 \%$ patients. Simple mastectomy was done in $6.7 \%$ patients. In the present study Post Mastectomy Immediate
Breast Reconstruction was done with LD flap in 53.3\% patients, LD flap with Silicone Implant in $20 \%$ patients and TRAM flap in $26.7 \%$ patients which is comparable to study conducted by Sarkar et al. ${ }^{[1]}$. In Present study, $18.18 \%$ patients who underwent LD flap reconstruction had marginal flap necrosis. Partial and Total flap necrosis was not observed in any of these patients. This is comparable to the study conducted by Shaikh et al. ${ }^{[3]}$ wherein Marginal Flap Necrosis was observed in $11 \%$ patients and Partial Flap Necrosis in $5.55 \%$ patients and Total Flap Necrosis in none with LD Flap. In the study conducted by Sarkar et al. [1] where Marginal Flap Necrosis, Partial Flap Necrosis and Total Flap Necrosis did not occur in any of the patients with LD Flap.

Patients in whom TRAM Flap reconstruction was done, $62.5 \%$ patients had Partial Flap Necrosis and $37.5 \%$ patients had Marginal Flap Necrosis, none had Total Flap Necrosis. This was only cuticular necrosis which recovered in 10 to 12 days. This is comparable to the study conducted by Sarkar et al. ${ }^{[1]}$ where Marginal Flap Necrosis was observed in $66.66 \%$ patients, Partial Flap Necrosis in $33.33 \%$ patients with no patients with Total Flap Necrosis. Similar study conducted by Shaikh et al. [3] showed Marginal Flap Necrosis observed in $3.40 \%$ patients and Partial Flap Necrosis in $0.57 \%$ patients and Total Flap Necrosis did not occur in any of the patients with TRAM Flap reconstruction. Among the 6 patients who underwent simple insertion of Silicone Implant with LD Flap, migration of implant was noted in 1 patient $(16.7 \%)$, where the implant had migrated 
to the axilla. Seroma at donor site was observed in $13.63 \%$ patients after LD flap. This is in comparison to study carried out by Spear SI et al. ${ }^{[5]}$ wherein $17.8 \%$ patients had seroma at donor site. Donor site infection after LD Flap reconstruction was noted in $9.1 \%$ patients.

This is in contrast to study conducted by Spear SI et al. [5], Bassiouny et al. ${ }^{[6]}$ and Hartrampf et al. ${ }^{[7]}$ wherein Donor Site infection was observed in none of the patients. Post TRAM Flap seroma at donor site was observed in none of the patients. This is in contrast to study performed by Hartrampf et al. ${ }^{[7]}$ where seroma at donor site was observed in $26 \%$ patients. Donor site infection after TRAM Flap was observed in $12.5 \%$ patients. This is in comparison with study carried out by Bassiouny et al ${ }^{[6]}$. It was observed in $12.5 \%$ patients and in study performed by Hartrampf et al. ${ }^{[7]}$ it was noted in $1.3 \%$ cases. Average hospital stay for LD Flap patients in the present study was 9 days. This is comparable with study carried out by Shaikh et al. ${ }^{[3]}$ wherein it was 7-8 days and in study conducted by Perdikis et al. ${ }^{\left[{ }^{[8]}\right.}$ it was 2 days. Average hospital stay in TRAM Flap patients was 14 days. This is in contrast to the study conducted by Shaikh et al. [3] where it was 7-8 days and in the study conducted by Hartrampf et al. ${ }^{[7]}$ it was 7.2 days. This was due to rural setup where patients have limited access to healthcare facilities close by. Recurrence of Breast carcinoma occurred in $6.7 \%$ patients who underwent Post Mastectomy Immediate Breast Reconstruction. This is contrast to the study carried by Hartrampf et al. ${ }^{[7]}$ where it was not noted in any of the patients. This is due to poor follow up of patients for adjuvant therapy.

Reconstructed breast was symmetrical with the opposite breast in 14 patients $(46.7 \%)$. In the remaining 16 patients, opposite side reduction mammoplasty was required in $10(33.3 \%)$ patients, in $5(16.7 \%)$ patients of TRAM Flap partial flap necrosis had occurred, and in one patient silicone implant migrated to the axilla. This is comparable to the study conducted by Hartrampf et al. ${ }^{[7]}$ where symmetry was achieved in $52 \%$ patients. $20 \%$ patients underwent nipple and areola reconstruction after 3 months of Breast reconstruction. This is in contrast with study carried by Shaikh et al. ${ }^{[3]}$ wherein $0.55 \%$ patients underwent nipple and areola reconstruction. 22 patients (73.3\%) were satisfied with Post Mastectomy Immediate Breast Reconstruction though symmetry of breast was achieved in 14 (46.7\%) patients, $10(33.3 \%)$ patients achieved shape but required opposite mammoplasty for symmetry but were not willing for the procedure as they were satisfied with Post Mastectomy Immediate Breast Reconstruction. 8(26.7\%) patients were not satisfied with the procedure because of Implant Migration which occurred in 1 patient and Partial Flap Necrosis happened in 5 patients, 2 patients had recurrence of carcinoma. All $6(100 \%)$ patients were satisfied after nipple and areola reconstruction.

\section{Conclusion}

Post Mastectomy Immediate Breast Reconstruction should be undertaken whenever possible. LD Flap reconstruction is simple to perform and safest technique of Breast Reconstruction. TRAM flap is cosmetically excellent but good only in expert hands. Nipple and areola reconstruction is final stage after immediate breast reconstruction is not mandatory and depends on patient's choice. Thus this study conclude that Post Mastectomy Immediate Breast Reconstruction can be feasible in rural set up.

\section{References}

1. Sarkar DK, Maji A, Saha S, Biswas JK. Oncoplastic Breast Surgery - Our Experiences in the Breast Clinic, IPGME\&R, Kolkata. Indian J Surg Oncol. 2011; 2(2):112-117.

2. Losee JE, Gimbel M, Rubin P, Wallace CG, Fu-Chan Wei. Plastic and reconstructive surgery. In: Brunicardi FC, Andersen DK, Hunter JG, Billiar TR, Matthews JB, Dunn DL, Pollock RE, editors. Schwartz's Principles of Surgery. 9thed. New York: Mc-Graw Hill Medical, 2010, 16821690.

3. Shaikh IAA, Thomas H, Vidyadharan R, Verghis V, Iype V, Abraham SJ. Post Mastectomy Immediate Breast Reconstruction: 13 Years' Experience in a Single Centre. Indian J Surg Oncol. 2010; 1(3):250-255.

4. Roehl KR, Wilhelmi BJ, Philips LG. Breast Reconstruction. In: Townsend CM, Beauchamp RD, Evers B M, Mattox KL, editors. Sabiston text book of surgery: The biological Basis of modern surgical practice. 19th ed. Philadelphia: Saunders. 2012; 1:870-883.

5. Spear SL, Boehmler JH, Taylor NS, Prada C. The role of the latissimus dorsi flap in reconstruction of the irradiated breast. Plast Reconstr Surg. 2007; 119(1):1-9.

6. Bassiouny MM, Maamoun SI, El-Shazly Sel-D. Youssef OZ.TRAM flap for immediate post mastectomy reconstruction: comparison between pedicled and free transfer. J Egypt Natl Canc Inst. 2005; 17(4):231-8.

7. Hartrampf CR, Bennett GK. Autogenous tissue reconstruction in the mastectomy patient. A critical review of 300 patients. Annals Surg. 1987; 205(5):508-519.

8. Perdikis G, Koonce S, Collis G, Eck D. Latissimus dorsi myocutaneous flap for breast reconstruction: bad flap or good flap?.Eplasty [Internet], 2011. [Cited 2011 Oct17]; 11: e39: [about6p.]. Available from: http://www.ncbi.nlm.nih.gov/pmc/articles/PMC3196917. 